EPJ Web of Conferences 64, 08005 (2014)

DOI: $10.1051 /$ epjconf/ 20146408005

(C) Owned by the authors, published by EDP Sciences, 2014

\title{
Emission line diagnostics for accretion and outflows in young very low-mass stars and brown dwarfs
}

\author{
B.Stelzer ${ }^{1, a}$, J.M.Alcalá ${ }^{2}$, E.Whelan ${ }^{3}$, and A.Scholz ${ }^{4}$ \\ ${ }^{1}$ INAF - Osservatorio Astronomico di Palermo, Piazza del Parlamento 1, 90134 Palermo, Italy \\ ${ }^{2}$ INAF - Osservatorio Astronomico di Capodimonte, Via Moiariello 16, 80131 Napoli, Italy \\ ${ }^{3}$ Institut für Astronomie \& Astrophysik, Eberhard-Karls-Universität Tübingen, 72076 Tübingen, Germany \\ ${ }^{4}$ School of Physics \& Astronomy, University of St. Andrews, The North Haugh, St. Andrews, Fife KY16 9SS, \\ United Kingdom
}

\begin{abstract}
We discuss accretion and outflow properties of three very low-mass young stellar objects based on broad-band mid-resolution X-Shooter/VLT spectra. Our targets (FU Tau A, 2M 1207-39, and Par-Lup3-4) have spectral types between M5 and M8, ages between $1 \mathrm{Myr}$ and $\sim 10 \mathrm{Myr}$, and are known to be accreting from previous studies. The final objective of our project is the determination of mass outflow to accretion rate for objects near or within the substellar regime as a probe for the T Tauri phase of brown dwarfs and the investigation of variability in the accretion and outflow processes.
\end{abstract}

\section{Introduction}

According to the paradigm of accretion in low-mass young stellar objects (YSOs) the inner-disk material is channeled along magnetic field lines onto the star [1] where it dissipates its kinetic energy in standing shocks [2]. Jets and molecular outflows are believed to be intimately related to the accretion process, carrying away the angular momentum. The ratio between mass outflow and mass accretion rate $\left(\dot{M}_{\text {out }} / \dot{M}_{\text {acc }}\right)$ is, therefore, a key parameter of jet launching models [3, 4]. For T Tauri stars values in a range of $\approx 0.01-0.1$ have been measured [5,6]. Only few estimates have been obtained for the faint end of the stellar sequence where preliminary results suggest the $\dot{M}_{\text {out }} / \dot{M}_{\text {acc }}$ ratio to be significantly higher (e.g. [7]). The sensitive latest generation spectrographs enable to extend studies of the accretion/outflow connection into the brown dwarf regime.

We present results of broad-band mid-resolution $(350-2500 \mathrm{~nm}, R \sim 9000) \mathrm{X}$-Shooter/VLT spectroscopy for three YSOs. The data were obtained in the framework of the Italian GTO Survey of nearby galactic star forming regions [8]. X-Shooter spectroscopy allows for a detailed characterization of YSOs, including an accurate assessment of their fundamental parameters, kinematics, rotation, and magnetic activity. It provides a rich database of accretion diagnostics from the $\mathrm{Br} \gamma$ and $\mathrm{Pa} \beta$ lines in the near-IR to the Balmer jump in the UV including the full optical band with the Balmer series and He $\lambda 5876$ and the Ca IRT. Finally, outflows can be traced through forbidden line emission.

In this contribution we focus on three of the most interesting individual objects from our survey. The characteristics and a motivation for their selection as X-Shooter targets are given in Sect. 2. We

a e-mail: stelzer@astropa.inaf.it

This is an Open Access article distributed under the terms of the Creative Commons Attribution License 2.0, which permits unrestricted use, distribution, and reproduction in any medium, provided the original work is properly cited. 
Table 1. Target properties and X-shooter observing log. Additional data for Par-Lup3-4 is described in Sect. 4.3.

\begin{tabular}{lccccccc}
\hline Name & Region & $\begin{array}{c}\text { distance } \\
{[\mathrm{pc}]}\end{array}$ & SpT & $\begin{array}{c}M_{*} \\
{\left[M_{\odot}\right]}\end{array}$ & $\begin{array}{c}R_{*} \\
{\left[R_{\odot}\right]}\end{array}$ & Obs.date & $\begin{array}{c}\text { Exp. } \\
{[\mathrm{s}]}\end{array}$ \\
\hline FU Tau A & Taurus & 140 & M6.5 & 0.08 & 1.4 & Jan 11, 2011 & 1800 \\
2M 1207-39 & TWA & 53 & M8 & 0.035 & 0.21 & Mar 22, 2010 / Apr 19, 2012 & 3600 \\
Par-Lup3-4 & Lupus III & 200 & M5 & 0.13 & 0.18 & Apr 7, 2010 & 3600 \\
\hline
\end{tabular}

describe the methods to derive mass accretion and outflow rates in Sect. 3. The results are presented in Sect. 4, conclusions and open questions are summarized in Sect. 5.

\section{Targets}

More than 80 YSOs were observed with X-Shooter in our survey of low-mass galactic star forming regions. Here we describe the results for three objects with particularly interesting or puzzling properties in the previous literature. The target list is presented in Table 1.

FU Tau A is the primary in a wide brown dwarf binary system with 5.7" separation corresponding to $\approx 800 \mathrm{AU}$ at the distance of Taurus [9]. Various signatures of youth include the presence of a circumstellar disk for both components inferred from near-IR excess emission and ongoing accretion inferred from $\mathrm{H} \alpha$ emission. The binary is located in the Barnard 215 dark cloud with no other young star nearby. Its isolation makes FU Tau a benchmark object for brown dwarf formation scenarios. FU Tau A appears well above the youngest isochrone of evolutionary pre-main sequence models in the HR diagram. FU Tau B, also appears younger than 1 Myr but by a smaller amount [10]. One objective for observing FU Tau A with X-Shooter was a better characterization of its accretion given the evidence for X-ray emission produced by an accretion shock from a Chandra observation [11].

2M1207-39 is the most well-studied among the brown dwarfs in the nearby $\approx 10$ Myr old TW Hya association. It has a disk from which it is accreting [12], an outflow [13] and a planetary companion [14]. An almost two orders of magnitude variation of the accretion rate has been inferred in the past based on the $\mathrm{H} \alpha$ emission from different epochs [15]. This strong variability and the perception of 2M 1207-39 as a kind of prototype accreting brown dwarf have led us to include it in our X-Shooter survey.

Par-Lup3-4 is a very low-mass star in the Lupus III cloud discovered by [16]. It is underluminous by $\approx 4$ mag with respect to other young M5 stars in the same star forming complex. This can be explained by an edge-on disk. In fact, a disk inclination angle of $\sim 81^{\circ}$ was inferred from SED modelling [17]. The jet of Par-Lup3-4 was imaged in optical forbidden lines [18]. Our multi-epoch $\mathrm{X}$-Shooter spectroscopy aims at studying the accretion and outflow variability and the relation of the mass accretion and mass outflow rates. The high inclination of the disk is believed to be favorable for the detection of variations related to non-azimuthal structures in the accretion geometry.

\section{Measurement of mass accretion and outflow rates}

We use three methods to measure mass accretion rates, $\dot{M}_{\text {acc }}$, from the X-Shooter spectra. First, we use the empirical relation with the full width of the $\mathrm{H} \alpha$ line at $10 \%$ of the peak height, $\log \dot{M}_{\text {acc }}\left[\mathrm{M}_{\odot} / \mathrm{yr}\right]=(-12.89 \pm 0.3)+(9.7 \pm 0.7) \cdot 10^{-3} \cdot W_{10 \%}[\mathrm{~km} / \mathrm{s}] ;$ see [19]. Secondly, we apply empirical correlations between the luminosity of individual emission lines $\left(L_{\text {line }}\right)$ and the accretion luminosity of the type $L_{\text {acc }}=a+b \cdot L_{\text {line }}$ where $a, b$ are coefficients that have been presented e.g. by [20]. These correlations have been newly calibrated for an unprecedentedly large number of lines 


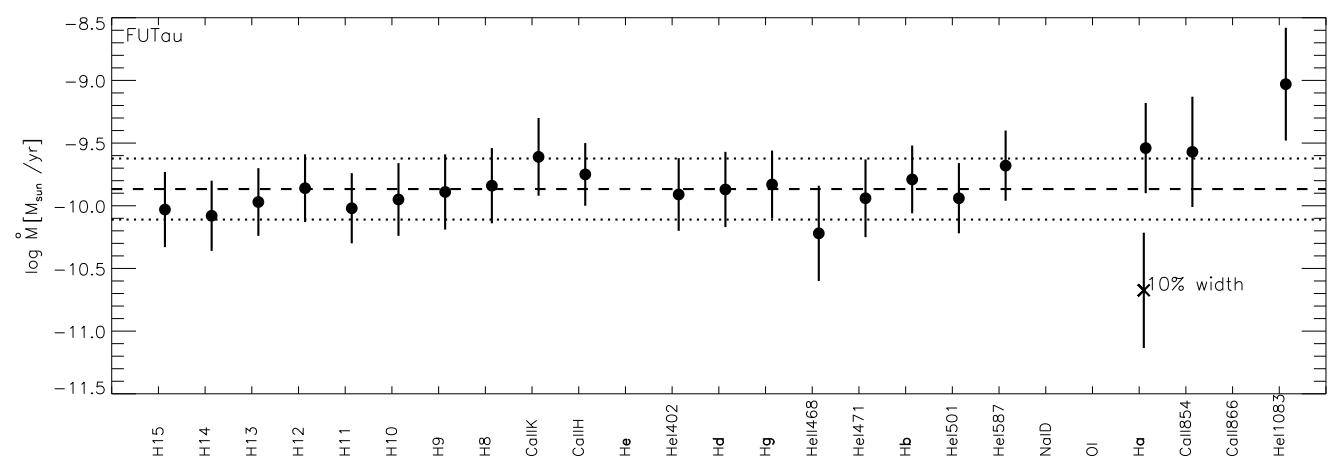

Figure 1. Mass accretion rate for FU Tau A derived from various emission line fluxes and luminosities using new calibrations from [21], and from the $\mathrm{H} \alpha 10 \%$ width. Dashed and dotted lines are mean and standard deviation for the luminosity-based $\dot{M}_{\text {acc }}$ excluding lines with suspected wind contribution, blending or uncertain calibration.

on the basis of the X-Shooter spectra of low-mass stars in Lupus obtained within our GTO program [21]. Finally, we measure the excess emission with respect to a purely photospheric spectrum. This is done by adding to the spectrum of a non-accreting template star, that has the same spectral type as the target, the emission of an isothermal slab model for a hot hydrogen gas. The model parameters (temperature, density and length of the slab) are adapted until the template plus model give the best fit to the target spectrum. This provides the accretion luminosity, $L_{\mathrm{acc}}$, which can be transformed into mass accretion rate according to $\dot{M}_{\text {acc }}=\left(1-1 / R_{\text {in }}\right)^{-1} \cdot \frac{L_{\text {acc }} R_{*}}{G M_{*}}$ where $R_{*}$ and $M_{*}$ are the stellar radius and mass, $G$ the gravitational constant, and $R_{\text {in }}$ the inner disk radius, assumed here to be $5 R_{*}$ (see [22]).

Outflow rates are measured from line luminosities using the prescription given by [5]. In particular, we use the relation $\log \dot{M}_{\text {out }}=X \cdot\left(1+\frac{n_{\mathrm{c}}}{n_{\mathrm{e}}}\right)\left(\frac{V_{\mathrm{T}}}{150 \mathrm{~km} / \mathrm{s}}\right)\left(\frac{l_{\mathrm{T}}}{2 \cdot 10^{15} \mathrm{~cm}}\right)^{-1}\left(\frac{L_{\text {line }}}{L_{\odot}}\right)$. Here, $n_{\mathrm{c}}$ is the ion-specific critical density and $n_{\mathrm{e}}$ the electron density. The projected aperture size on the plane of the sky $\left(l_{T}\right)$ is determined by the slit width and the distance to the target. The tangential velocity $\left(V_{T}\right)$ can be obtained from the measured radial velocity if the inclination angle of the outflow is known. $X$ is a numerical constant calculated by [5] for the most prominent forbidden lines.

\section{Results}

\subsection{FU Tau A}

In Fig. 1 we show the values for $\dot{M}_{\text {acc }}$ that we obtain from the individual emission line luminosities using the calibrations of [21] and from the $\mathrm{H} \alpha 10 \%$ width applying the relation from [19]. While the various line luminosities yield consistent values within the uncertainties, the width of $\mathrm{H} \alpha$ results in a lower estimate for $\dot{M}_{\text {acc }}$. From the slab model we obtain a third value for $\dot{M}_{\text {acc }}$. That latter estimate is $\log \dot{M}_{\text {acc }}\left[\mathrm{M}_{\odot} / \mathrm{yr}\right]=-10.1$ and it agrees well with the result from the line luminosities. The discrepancy of the value obtained from the $\mathrm{H} \alpha$ width supports previous notions of the poor reliability of this tracer as quantitative measure for accretion (e.g. [20, 21, 23]).

We have found for the first time outflow activity in FU Tau A, detected in the form of forbidden emission lines, and we use the [OI] $\lambda 630.0 \mathrm{~nm}$ and [SII] $\lambda 673.1 \mathrm{~nm}$ emission to derive the mass loss rate, $\dot{M}_{\text {out }}$. The values of $\dot{M}_{\text {out }}\left(\approx 4 \cdot 10^{-11} \mathrm{M}_{\odot} / \mathrm{yr}\right)$, and consequently $\dot{M}_{\text {out }} / \dot{M}_{\text {acc }} \approx 0.3$, are not well constrained because of uncertainties in the electron density and the inclination angle required 


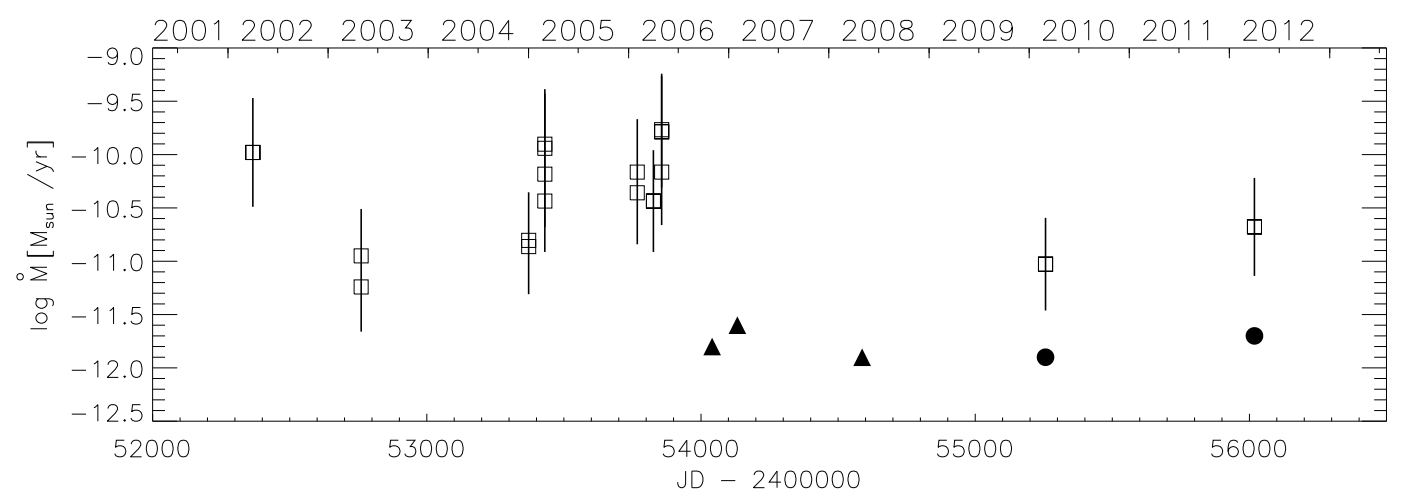

Figure 2. Mass accretion rate of 2M 1207-39 measured throughout the last decade with different methods: line luminosity (filled circles), modelling of UV excess (filled triangles) and $\mathrm{H} \alpha 10 \%$ width (open squares). For the latter one the plotting symbols denote the highest and the lowest values measured in the respective month and the vertical bars include the uncertainties of the conversion to mass accretion rate. The two most recent epochs shown in this graph are from our X-Shooter observations.

to calculate the tangential velocity. Specifically, as a result of the non-detection of [S II] $6617.7 \mathrm{~nm}$ emission only a lower limit can be determined for $n_{\mathrm{e}}$. Furthermore, the disk inclination angle required to calculate $V_{T}$ is also a lower limit, $i_{\text {disk }} \geq 50^{\circ}$ from a comparison of the measured $v \sin i$ and the rotation period observed photometrically by [24]; see also [25] for details. The outflow of FU Tau A has recently also been detected at millimeter wavelengths [26], and this estimate of the molecular outflow rate is an order of magnitude higher than that obtained from the atomic transitions.

\section{$4.22 \mathrm{M} 1207-39$}

2M 1207-39 is one of the best-studied young accreting brown dwarfs. We have collected accretion measurements from the literature. The resulting time-series for $\dot{M}_{\mathrm{acc}}$ is shown in Fig. 2. Different plotting symbols refer to different techniques for calculating the accretion rate (see figure caption). Our X-Shooter observations are represented by the two most recent epochs. It can be noticed that all values derived from the $\mathrm{H} \alpha$ width yield systematically higher $\dot{M}_{\text {acc }}$ than other methods (UV excess modelling and line luminosity). Our simultaneous measurement of $\mathrm{H} \alpha$ width and line luminosities (the two most recent epochs in Fig. 2) prove that there are discrepancies associated with the different analysis methods which, if ignored, could be mistaken for true accretion variations. As noted above for the case of FU Tau A UV excess and line luminosity are considered more reliable quantitative accretion diagnostics. All data points obtained so far for 2M 1207-39 from these two tracers suggest at most modest variability of the accretion rate on time-scales of years.

\subsection{Par-Lup3-4}

The mass accretion rates of Par-Lup3-4 derived from the line luminosities with the calibrations from [21] are shown in the left panel of Fig. 3. The actual measurements (shown in black) need to be corrected for the grey extinction induced by the edge-on disk of Par-Lup3-4. Assuming that the same obscuration factor $(O F)$ suppresses both the bolometric luminosity and the accretion luminosity, $\dot{M}_{\text {acc }}$ 

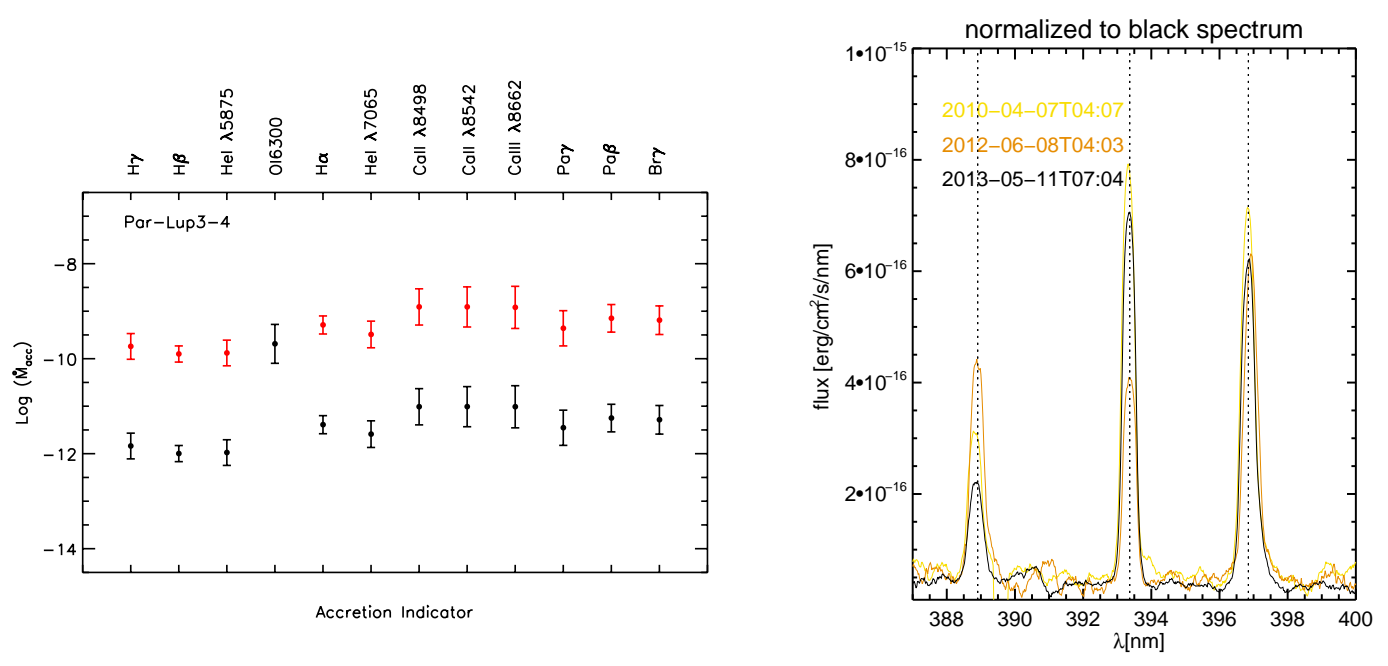

Figure 3. Results from X-Shooter spectroscopy of Par-Lup3-4: (Left) - Mass accretion rates calculated from line luminosities with the calibrations of [21] (black symbols) and same measurements corrected for the grey extinction induced by the edge-on disk (red symbols); see [27] for details. (Right) - Three different epochs showing apparently uncorrelated changes in the emission line fluxes. Shown are the spectrum obtained in the GTO program (yellow) and two spectra from our follow-up programs carried out in June 2012 and May 2013.

can be corrected according to $\left(\dot{M}_{\text {acc }}\right)_{\text {corr }}=O F^{1.5} \cdot \dot{M}_{\text {acc }}$. This is based on the relation between accretion rate and luminosity given in Sect. 3 and considers the $R_{*}^{2}$ proportionality of the bolometric luminosity; see [27] for more details. The corrected values for $\dot{M}_{\text {acc }}$ are shown in red in Fig. 3. No obscuration correction is applied to the [OI] $\lambda 630 \mathrm{~nm}$ emission because forbidden lines are generated in outflows not in accretion streams. The good agreement between the $\dot{M}_{\text {acc }}$ derived from [OI] $\lambda 630 \mathrm{~nm}$ with the extinction corrected values obtained for the other lines confirms (1) that the outflow is not obscured by the disk and (2) that there is a tight connection between outflow and accretion activity.

In June 2012 and May 2013 we obtained in total nine additional X-Shooter spectra of Par-Lup3-4 with the aim of studying its emission line variability. Thus, the available spectra for this object cover timescales from few hours to three years. Our preliminary analysis shows evidence for changes in the flux distribution from years 2010 to 2013, e.g. the June 2012 spectrum displays the strongest Balmer H8 line but the weakest Ca II H\&K lines in Fig. 3. The changes in emission line flux are accompanied by variations in the spectral slope (not shown). A detailed investigation of all available spectra will shed light on variations on different timescales.

\section{Conclusions and open questions}

For all our observations, but particularly demonstrated for 2M 1207-39, the $\mathrm{H} \alpha 10 \%$ width yields mass accretion rates that are inconsistent with results from other methods, possibly due to the influence of outflows on the line profiles. The case of Par-Lup-3-4 shows the importance of an accurate assessment of extinction for measuring $\dot{M}_{\text {acc }}$ and, consequently, the mass inflow-to-outflow ratio. For the example of FU Tau A we discuss the difficulties in obtaining accurate $\dot{M}_{\text {out }}$ estimates from forbid- 
den lines. Future sensitive observations of larger samples of very low-mass objects will also have to address the discrepancies between outflow rates determined from atomic and molecular transitions.

\section{References}

[1] A. Koenigl, ApJL 370, L39 (1991)

[2] N. Calvet, E. Gullbring, ApJ 509, 802 (1998)

[3] R.E. Pudritz, R. Banerjee, The disc-jet connection, in Massive Star Birth: A Crossroads of Astrophysics, edited by R. Cesaroni, M. Felli, E. Churchwell, M. Walmsley (2005), Vol. 227 of IAU Symposium, pp. 163-173, arXiv: astro-ph/0507268

[4] J. Ferreira, C. Dougados, S. Cabrit, A\&A 453, 785 (2006), arXiv: astro-ph/0604053

[5] P. Hartigan, S. Edwards, L. Ghandour, ApJ 452, 736 (1995)

[6] S.Y. Melnikov, J. Eislöffel, F. Bacciotti, J. Woitas, T.P. Ray, A\&A 506, 763 (2009), 0910. 0379

[7] E.T. Whelan, T.P. Ray, L. Podio, F. Bacciotti, S. Randich, ApJ 706, 1054 (2009), 0910. 2665

[8] J.M. Alcalá, B. Stelzer, E. Covino, G. Cupani, A. Natta, S. Randich, E. Rigliaco, L. Spezzi, L. Testi, F. Bacciotti et al., Astronomische Nachrichten 332, 242 (2011)

[9] K.L. Luhman, E.E. Mamajek, P.R. Allen, A.A. Muench, D.P. Finkbeiner, ApJ 691, 1265 (2009a), 0902.0425

[10] K.L. Luhman, E.E. Mamajek, P.R. Allen, A.A. Muench, D.P. Finkbeiner, ApJ 720, 1781 (2010)

[11] B. Stelzer, A. Scholz, C. Argiroffi, G. Micela, MNRAS 408, 1095 (2010), 1006. 2717

[12] A. Scholz, R. Jayawardhana, A. Brandeker, ApJL 629, L41 (2005), astro-ph/0507122

[13] E.T. Whelan, T.P. Ray, F. Comeron, F. Bacciotti, P.J. Kavanagh, ApJ 761, 120 (2012), 1210.7106

[14] G. Chauvin, A.M. Lagrange, C. Dumas, B. Zuckerman, D. Mouillet, I. Song, J.L. Beuzit, P. Lowrance, A\&A 425, L29 (2004), astro-ph/0409323

[15] B. Stelzer, A. Scholz, R. Jayawardhana, ApJ 671, 842 (2007), arXiv: 0707 . 1754

[16] F. Comerón, M. Fernández, I. Baraffe, R. Neuhäuser, A.A. Kaas, A\&A 406, 1001 (2003)

[17] N. Huélamo, H. Bouy, C. Pinte, F. Ménard, G. Duchêne, F. Comerón, M. Fernández, D. Barrado, A. Bayo, I. de Gregorio-Monsalvo et al., A\&A 523, A42 (2010), 1008. 5065

[18] M. Fernández, F. Comerón, A\&A 440, 1119 (2005), astro-ph/0506307

[19] A. Natta, L. Testi, J. Muzerolle, S. Randich, F. Comerón, P. Persi, A\&A 424, 603 (2004), arXiv: astro-ph/0406106

[20] G.J. Herczeg, L.A. Hillenbrand, ApJ 681, 594 (2008), 0801. 3525

[21] J.M. Alcalá, A. Natta, C.F. Manara, L. Spezzi, B. Stelzer, A. Frasca, K. Biazzo, E. Covino, S. Randich, E. Rigliaco et al., A\&A submitted (2013)

[22] E. Gullbring, L. Hartmann, C. Briceno, N. Calvet, ApJ 492, 323 (1998)

[23] G. Costigan, A. Scholz, B. Stelzer, T. Ray, J.S. Vink, S. Mohanty, MNRAS 427, 1344 (2012), 1209.0462

[24] A. Scholz, B. Stelzer, G. Costigan, D. Barrado, J. Eislöffel, J. Lillo-Box, P. Riviere-Marichalar, H. Stoev, MNRAS 419, 1271 (2012), 1109.3474

[25] B. Stelzer, J.M. Alcalá, A. Scholz, A. Natta, S. Randich, E. Covino, A\&A 551, A106 (2013), 1301.0410

[26] J.L. Monin, E.T. Whelan, B. Lefloch, C. Dougados, C. Alves de Oliveira, A\&A 551, L1 (2013), 1301.4387

[27] E. Whelan, R. Bonito, S. Antoniucci, J.M. Alcalá, T. Giannini, B. Nisini, F. Bacciotti, L. Podio, B. Stelzer, F. Comerón, A\&A submitted (2013) 